\title{
Influencia del vidrio, azufre y sales de metales alcalinos en la porosidad del aluminio reciclado
}

\author{
Jenny Esmeralda Padilla ${ }^{1}$ \\ Covadonga Álvarez Maldonado ${ }^{2}$
}

\section{RESUMEN}

Los estudiantes del módulo de Metalurgia Aplicada de la Carrera de Química Industrial de la UNAH-VS del primer período de 2014, desarrollaron una investigación con el fin de buscar una mejora en la porosidad que presenta el aluminio reciclado de latas para diferentes tipos de bebidas.

Para el desarrollo de este estudio se tomó como punto de partida la baja producción de aluminio que existe en nuestro país, siendo esta generada únicamente por procesos de fundición artesanales. Sin embargo, durante estos procesos el aluminio obtenido presenta baja dureza y, por tanto, un alto grado de porosidad, lo que impide la comercialización del aluminio reciclado en el extranjero. Es por ello que se decidió buscar una composición química adecuada para la producción de aluminio de alta dureza a partir del reciclaje de latas de este metal.

Con el fin de llevar a cabo los diferentes experimentos, se fabricó un horno de fundición para elaborar diferentes aleaciones de aluminio conteniendo: azufre, vidrio, sal de mesa y bicarbonato de sodio en diversas combinaciones y proporciones; posteriormente, se realizó una determinación de sus densidades y durezas.

Se hizo una comparación de las durezas y densidades obtenidas y se identificó una composición química que permita disminuir la porosidad del aluminio obtenido por reciclaje, con lo cual se espera obtener aluminio con un alto grado de pureza mediante la utilización de sales de metales alcalinos como fundente y aplicando una producción más limpia en la medida de lo posible.

\footnotetext{
${ }^{1}$ Estudiante, Carrera de Química Industrial. Departamento de Química UNAH-VS. San Pedro Sula, Honduras.: jennypadso@hotmail.com

${ }^{2}$ Asesora, Profesora, Carrera de Química Industrial. Departamento de Química, UNAH-VS. San Pedro Sula, Honduras.: covadonga.alvarez@unah.edu.hn
} 
Palabras clave: reciclaje del aluminio, porosidad, dureza Brinell.

\section{ABSTRACT}

The Applied Metallurgy course students of industrial Chemistry Career performed a research to look for an improvement in the Aluminum material porosity melted from recycled aluminum cans of soda drinks and others.

In order to develop the study, it was necessary to take as starting point the low aluminum production that exists in our country, being only generated by artesian melting processes. Nevertheless, during these processes, the aluminum produced shows low hardness and therefore high porosity. This prevents the commercialization of the recycled aluminum in foreign countries. That is why we have decided to look for an adequate chemical composition to produce high hardness aluminum from the recycled cans.

Due to this objective a melting oven was made and its purpose was to develop different aluminum alloys containing sulfur, glass, table salt and baking soda in different compositions, and then after to measure their densities and hardness.

A comparison was performed for the different hardness and densities against the chemical composition and the porosity of the material produced from the recycling process. On the other hand, another objective is to get of high purity using different compositions of alkaline metals salts as smelter and applying a clean production technology into the process.

Keywords: recycled aluminum, porosity, Brinell hardness. 


\section{INTRODUCCIÓN}

El aluminio es un metal ampliamente utilizado debido a sus excelentes propiedades, las que incluyen resistencia mecánica, alta conductividad térmica y eléctrica, ligereza y resistencia a la corrosión; además, su bajo costo en comparación con otros metales y su abundancia, hacen de este un metal indispensable para el desarrollo de la sociedad. Sin embargo, su producción requiere un alto costo y no existe ninguna industria extractora de aluminio a partir de la bauxita en el país.

Este hecho obliga a importar todo el aluminio de buena calidad que Honduras demanda. Debido a ello, han surgido en el país muchas recicladoras de aluminio, tomando ventaja de la capacidad del mismo metal para poder reciclarlo indefinidas veces sin disminuir significativamente su calidad.

No obstante, la mayoría de estas recicladoras se basan únicamente en la fusión de chatarra de aluminio, como latas y otras piezas, y en su consecuente solidificación, prestando poco o nada de atención a la composición química y propiedades del producto final. Esto ocasiona que, en su mayoría, el aluminio que se obtiene del reciclaje de latas en el país, sea poroso y poco resistente a la tensión, de baja dureza y, por tanto, quebradizo, por lo cual no puede ser utilizado en la fabricación de piezas que requieran que el mismo posea una alta calidad.

Es por ello que se decidió realizar una investigación para determinar una composición química adecuada para que el aluminio que se pueda obtener a partir del reciclaje de latas que se comercializan en Honduras posea mejores características, tales como un menor grado de porosidad y, en consecuencia, una mayor dureza y densidad. Así, se pretendió evaluar la influencia del vidrio, azufre y sales de metales alcalinos en el aluminio reciclado a partir de latas de bebidas.

\section{Generalidades}

El aluminio es un metal blanco plateado, relativamente blando y que pulido semeja a la plata. Cristaliza en red cúbica centrada en las caras (FCC). Su peso específico es igual a 2,699, es decir, casi $1 / 3$ del hierro $(7,87)$. El único metal industrial más ligero que el aluminio es el magnesio, de peso específico 1,74. Su conductividad eléctrica es un $60 \%$ de la del cobre y 3,5 veces mayor que la del hierro. Su punto de fusión es $660^{\circ} \mathrm{C}$ y el de ebullición $2.450^{\circ} \mathrm{C}$. Este punto de fusión relativamente bajo, unido a su punto de ebullición bastante alto facilita su fusión y moldeo. [6] 


\section{Aleaciones de aluminio}

Se denominan aleaciones ligeras a aquellas aleaciones que tienen como elemento base o principal el aluminio.

Aparte sus aplicaciones eléctricas, la mayoría del aluminio se emplea en forma de aleaciones. Éstas poseen una resistencia mecánica mayor que la del aluminio puro, conservando por otra parte, las ventajas del poco peso, buena conductividad y resistencia a la corrosión. [5]

Los principales metales empleados para su aleación con aluminio son los siguientes: Cobre (Cu), silicio (Si), cinc ( $\mathrm{Zn})$, magnesio (Mg), y manganeso (Mn). Y los que pudiéramos considerar como secundarios, son los siguientes: Níquel (Ni), titanio (Ti), hierro $(\mathrm{Fe})$, cromo $(\mathrm{Cr})$ y cobalto (Co). Sólo en casos especiales se adicionan: Plomo (Pb), cadmio $(\mathrm{Cd})$, antimonio $(\mathrm{Sb})$ y bismuto $(\mathrm{Bi})$. En general, la proporción total en que forman parte de las aleaciones ligeras, no pasa del 15\%. [6]

El aluminio es completamente inalterable en el aire, pues se recubre de una delgada capa de óxido, llamada alúmina, de algunas centésimas de micra, que protege el resto de la masa de la oxidación. Se trata de una capa refractaria, es decir, que posee una temperatura de fusión muy elevada por lo que se debe retirar antes o durante la operación de soldeo para permitir una buena fusión del metal base. Es debido a la formación de alúmina, que el aluminio es conocido por su resistencia a la corrosión frente al aire, agua, aceites, alimentos y muchos agentes químicos. Además, gracias a esta película protectora, resiste también a la acción del vapor de agua, el ataque nítrico concentrado y muchos otros compuestos químicos. En cambio, es atacado por el ácido sulfúrico, el clorhídrico, el nítrico diluido y las soluciones salinas.

\section{Fundición del aluminio}

En las aleaciones de aluminio, en presencia de aire, se forma alúmina (óxido de aluminio) con gran facilidad. Este óxido tiene una temperatura de fusión muy elevada, entre $1200^{\circ} \mathrm{C}$ y $2000^{\circ} \mathrm{C}$ mayor que la temperatura de fusión del aluminio. Por tanto el aluminio funde antes que su óxido y, cuando esto sucede, la película de óxido impide la fusión entre el metal base y el metal de aportación, por lo que es imprescindible eliminar o retirar la capa de óxido mediante un decapado químico, un fundente, amolado o mediante la acción decapante del arco eléctrico. 


\section{Reciclaje del aluminio}

El reciclaje del aluminio es un proceso mediante el cual, los desechos de aluminio pueden ser convertidos en otros productos tras su utilidad primaria. Este proceso implica simplemente refundir el metal. Las ventajas del reciclaje del aluminio son:

a) Al utilizar aluminio reciclado se ahorra un $95 \%$ de la energía empleada a partir de la producción del mineral primario.

b) Puede reciclarse indefinidamente sin perder sus propiedades, pudiéndose fabricar un producto con idénticas propiedades.

c) Se puede reciclar el $100 \%$ de los materiales recuperados. • Su recuperación es rentable técnica y económicamente.

d) En el vertedero ocupa espacio, no se degrada y es irrecuperable.[12]

\section{METODOLOGÍA}

Esta investigación es de nivel descriptivo, ya que se busca especificar propiedades y características de las piezas de aluminio. Se combinó una serie de elementos que permitieran mejorar la calidad del mismo. Es también de diseño experimental y de corte transversal. Se tomaron las siguientes variables para la elaboración de las hipótesis:

\begin{tabular}{|l|l|l|}
\hline No. & \multicolumn{1}{|c|}{ Variables independientes } & \multicolumn{1}{c|}{ Variables dependientes } \\
\hline 1 & Concentración de silicio proveniente del vidrio. & $\begin{array}{l}\text { Grado de dureza del aluminio obtenido } \\
\text { en el proceso. }\end{array}$ \\
\hline 2 & $\begin{array}{l}\text { Utilización de cloruro de sodio y de potasio } \\
\text { como fundentes durante el proceso de } \\
\text { reciclado de aluminio por fusión. }\end{array}$ & $\begin{array}{l}\text { Pureza del aluminio obtenido en el } \\
\text { proceso. }\end{array}$ \\
\hline 3 & Concentración de azufre. & Grado de dureza del aluminio obtenido. \\
\hline
\end{tabular}




\section{Descripción del proceso de obtención de las aleaciones y determinación de sus propiedades}

Se utilizaron latas de gaseosas, cervezas, té frío y bebidas energéticas. Estas latas, fabricadas en su mayor parte de aluminio, se comprimieron individualmente por impacto. Una vez comprimidas, se introdujeron diez latas a un crisol. Posteriormente, este se colocó dentro del recinto refractario, directamente sobre el carbón en combustión hasta que las latas se fundieran totalmente. El proceso de fundición fue realizado en el domicilio de uno de los integrantes del equipo.

La pieza se dejó solidificar, proceso que tardó en promedio de 2 a 3 minutos. La pieza sólida y fría se retiró del molde y se prosiguió con la siguiente colada. Durante todo el proceso se mantuvo un nivel casi constante de latas en el crisol, así como de carbón dentro del recinto refractario, lo cual dio como resultado la obtención de 7 piezas de diferentes composiciones.

Como componentes de las aleaciones se utilizaron cuatro sustancias: azufre en polvo, sal de mesa, vidrio y bicarbonato de sodio. La sal de mesa utilizada fue una mezcla de cloruro de potasio, yoduro de potasio y cloruro de sodio; conteniendo, además, silicato de calcio y dextrosa. El vidrio utilizado se adquirió a partir de una botella, sin colorantes, de bebida alcohólica; este material se trituró hasta la obtención de un fino polvo.

Con el objetivo de evaluar el efecto de su concentración producido en la dureza del aluminio obtenido, se elaboraron dos aleaciones únicamente con azufre, una al $1 \%$ y la otra al $4.98 \%$. Otras dos piezas fueron fabricadas mediante la adición de vidrio y sal para verificar el efecto que el silicio presente en el vidrio tiene sobre la dureza del aluminio y comprobar que la adición de sal de mesa ( $\mathrm{NaCl}$ ) como fundente puede ser beneficiosa si se usa en conjunto. Una de las piezas se fabricó con una concentración de vidrio del $4.96 \%$ y sal del $2.48 \%$. La segunda pieza estaba compuesta por una mezcla de sal al $2.02 \%$, vidrio al $1.02 \%$ y el resto de aluminio.

Se elaboró una única pieza conteniendo vidrio al $5.06 \%$ para observar el efecto del vidrio únicamente $y$, como un blanco, se dejó una de las piezas sin alear; esta solamente contenía aluminio proveniente del reciclaje de las latas. Para la elaboración de las aleaciones, inicialmente se fabricaron diversas piezas de aluminio sin alear, se midió la masa de cada una de estas piezas en una balanza granataria (0 balanza de precisión) y se fundieron nuevamente añadiendo en esta etapa las sustancias correspondientes y previamente pesadas para cambiar su composición. 
Determinación de densidades

Las determinaciones de densidad fueron realizadas en los laboratorios de química de la UNAH-VS. Para evaluar las densidades de las piezas obtenidas se utilizó un método basado en la medida de la masa del fluido desplazado por un cuerpo (principio de Arquímedes). En este caso, el cuerpo fue la pieza de aluminio a determinar y el fluido fue el agua a $27^{\circ} \mathrm{C}$, cuya densidad se consideró de $1 \mathrm{~g} / \mathrm{ml}$ por simplicidad. Luego se determinó la dureza Brinell de cada una de las piezas en las instalaciones del INFOP (Instituto Nacional de Formación Profesional).

\section{RESULTADOS Y ANÁLISIS}

A raíz de las pruebas de densidad y dureza a las que fueron sometidas cada una de las siete piezas elaboradas en el proceso de fundición, se obtuvieron los resultados que se presentan en los gráficos 1 y 2 . En estos aparecen las densidades y durezas Brinell de las aleaciones elaboradas, de la pieza de aluminio sin alear, de una pieza comercial de duraluminio y de las partes de una lata de cerveza de las mismas que se utilizaron en el proceso de reciclaje.

Las densidades de las piezas elaboradas por el equipo de investigación oscilan alrededor de $2.6 \mathrm{~g} / \mathrm{ml}$. Una comparación de las diferentes densidades de cada pieza elaborada se observa en el gráfico 1, en el cual puede observarse que la de mayor densidad es la 4B y la de menor densidad es la pieza AB (Ver Tabla1).

La pieza $A B$ con una composición de $100 \%$ de aluminio y una densidad de $2.32 \mathrm{~g} / \mathrm{ml}$, no sufrió cambios intencionales significativos en su composición, considerándose como no aleada. La densidad de la pieza no aleada es un indicativo del nivel de pureza que se puede obtener con el proceso de fundición llevado a cabo. La densidad de esta pieza difiere en un $14.07 \%$ con la densidad teórica del aluminio.

La segunda pieza con menor densidad fue la pieza 3B, con una composición del $94.94 \%$ de aluminio y un $5.06 \%$ de vidrio. Esta pieza resultó con una densidad de $2.54 \mathrm{~g} / \mathrm{ml}$. 


\section{Gráfico 1. Densidades de las piezas de aleación de aluminio elaboradas}

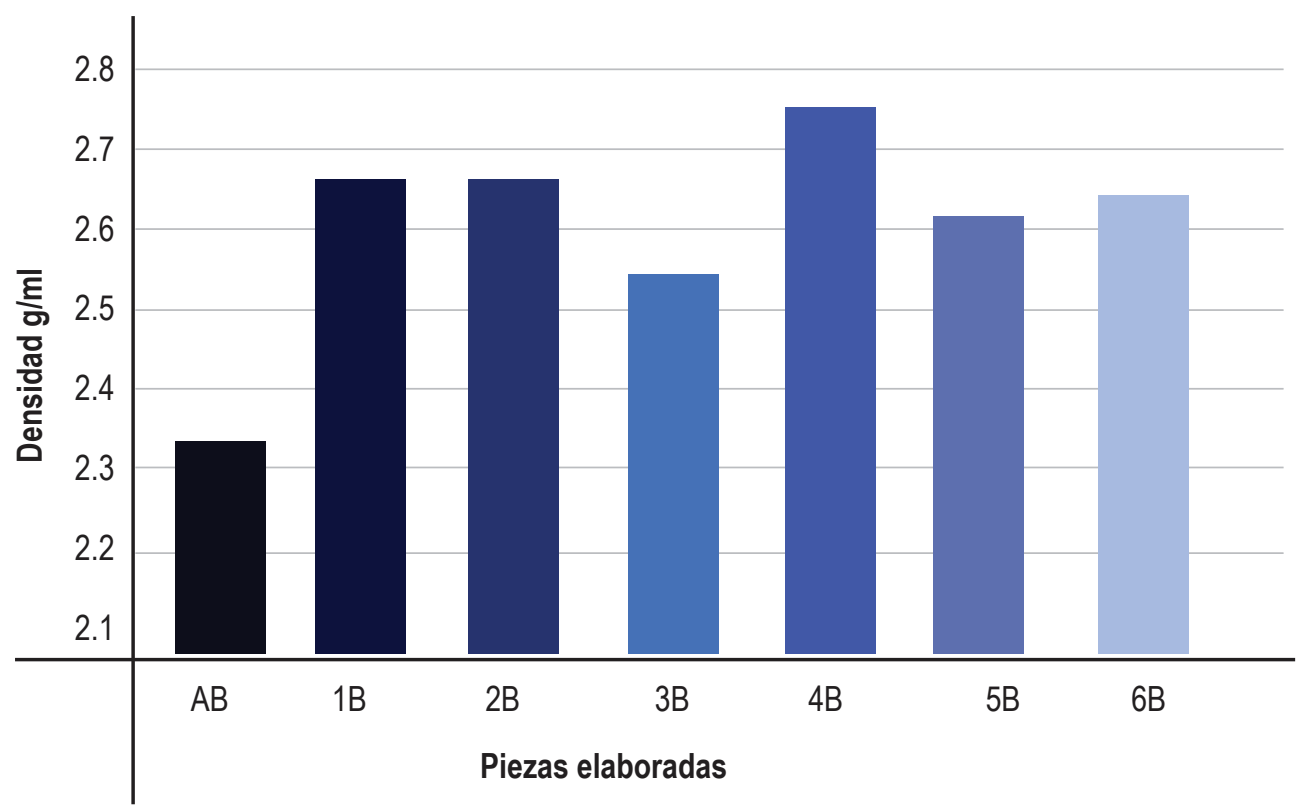

La pieza que resultó con la mayor densidad fue la denominada 4B, compuesta por un $99 \%$ de aluminio y $1 \%$ de azufre. La densidad de esta pieza metálica fue de 2.72 $\mathrm{g} / \mathrm{ml}$, muy cercana a la densidad teórica del aluminio puro, difiriendo solamente en un $0.74 \%$. La segunda pieza con la mayor densidad fue la 2B, presentando una densidad de $2.65 \mathrm{~g} / \mathrm{ml}$ y con una diferencia del $1.85 \%$ con respecto a la teórica. Las demás piezas presentaron densidades intermedias. Cabe destacar que cada una de las pruebas se realizó con el mayor nivel de precisión posible, a pesar de que se llevó a cabo una sola determinación para cada pieza.

En adición, se determinó la densidad de cada una de las partes de una lata de cerveza, con el fin de hacer una comparación entre las densidades de las piezas elaboradas (el producto final) y las latas de aluminio utilizadas (la materia prima del proceso). Este procedimiento brindó resultados relativamente alejados de la teoría, por lo que con él se confirma que ninguna parte de la lata de aluminio está fabricada en aluminio puro y que, de hecho, el nivel de impureza en la misma es alto.

El gráfico 2 representa los valores de dureza obtenida en cada una de las 7 piezas elaboradas, pudiendo observarse que la pieza que presentó una mayor dureza fue la pieza 6B, compuesta en un $95.02 \%$ de aluminio y un $4.98 \%$ de azufre, con un valor 
de dureza de 60.4 HB. Como parangón se utilizará la dureza que presentó una pieza de duraluminio, la cual fue de 109.34 HB. Así, se obtiene que la pieza más dura que se elaboró difiere en un $44.76 \%$, con respecto a la dureza de una de las aleaciones de aluminio más conocidas por sus excelentes propiedades mecánicas.

La segunda pieza más dura fue la 1B, con un valor de dureza de 55.37 HB. Esta pieza presentó una composición del $96.96 \%$ de aluminio, $2.02 \%$ de sal de mesa refinada y $1.02 \%$ de vidrio. Otra de las piezas con una dureza relativamente alta fue la denominada 4B, compuesta en un $99 \%$ de aluminio y $1 \%$ de azufre, con una dureza de $53.3 \mathrm{HB}$.

Las durezas más bajas obtenidas fueron en las piezas 3B (94.94 \% de aluminio y 5.06 $\%$ de vidrio) con una dureza de $34.6 \mathrm{HB}$ y $2 \mathrm{~B}$ (92.56 \% aluminio, $4.96 \%$ vidrio y 2.48 $\%$ sal de mesa) con una dureza de 41.93 .

La dureza de la pieza no aleada fue de 50.8 , un valor de dureza intermedio entre todas las aleaciones. De la misma manera, un valor de dureza muy cercano al valor promedio fue el de la pieza aleada con bicarbonato de sodio al $5.06 \%$ (5B).

\section{Gráfico 2. Durezas Brinell de las piezas de aleación de aluminio elaboradas}

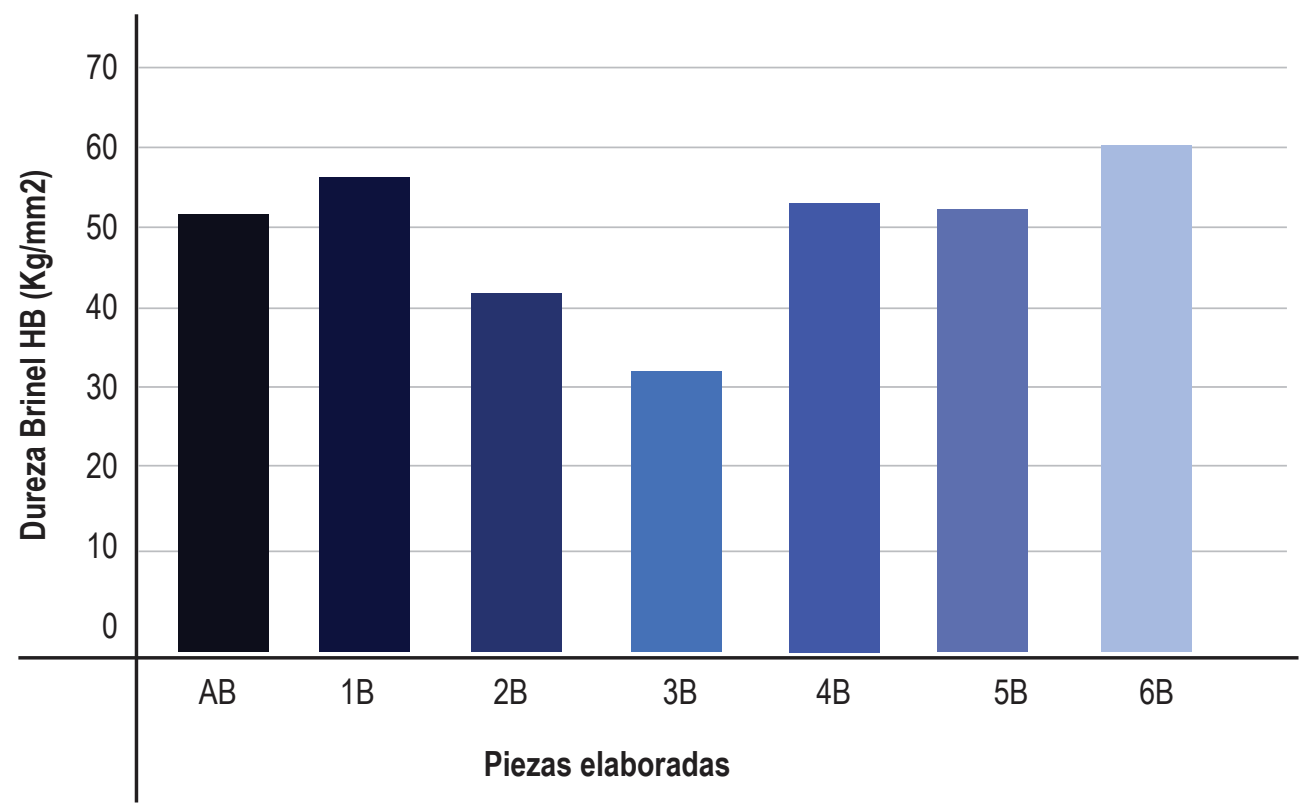


El gráfico 3 muestra las diferentes densidades de las partes de una de las latas de cerveza que se utilizó durante la fundición del aluminio. Al determinar la densidad de la anilla de la lata, se obtuvo una densidad de $1.3795 \mathrm{~g} / \mathrm{ml}$, una densidad mucho menor a la del aluminio puro. Esta densidad difiere en un $48.9 \%$ con el valor teórico. Por otra parte, la densidad de la parte superior de la lata, para cuya determinación se tomó la lámina desprendible del sistema de apertura de la misma, fue de $1.8940 \mathrm{~g} / \mathrm{ml}$.

\section{Gráfico 3. Densidades de las partes de una de las latas de cerveza utilizadas}

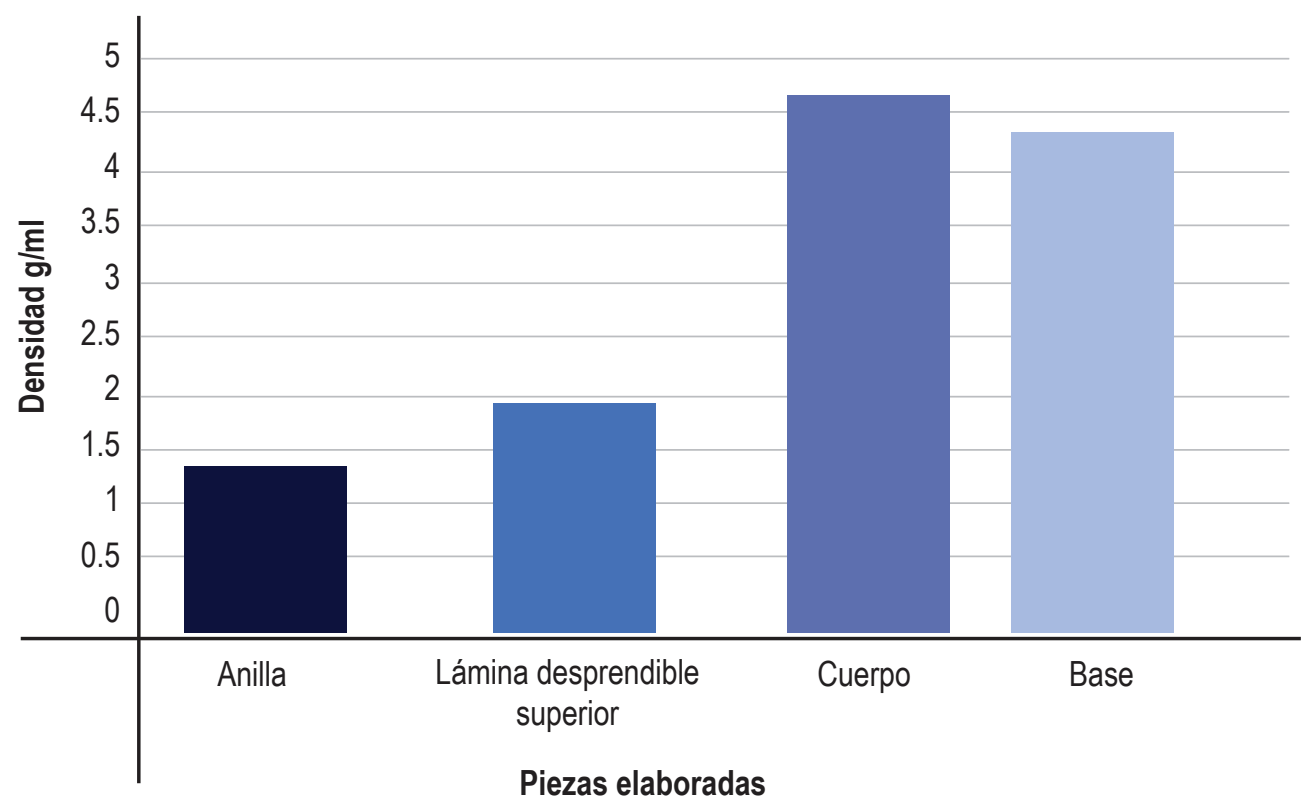

La diferencia de este valor con respecto al teórico es del $29.85 \%$, menos que la anilla de la lata. Este hecho lleva a deducir que la parte superior de la lata es más rica en aluminio que la anilla. La densidad del cuerpo de la lata de cerveza fue de $4.64 \mathrm{~g} / \mathrm{ml}$. Este alto valor difiere en un $71.85 \%$ con el valor de densidad teórico para el aluminio puro y lo cual podría indicar una alta dureza para esta parte de la lata.

Este análisis corresponde con la realidad, ya que el cuerpo es una de las partes de la lata que necesitan mayor resistencia mecánica.

De la misma manera, la densidad que se determinó a la base de la lata de cerveza fue bastante alta con respecto al valor teórico. Esta densidad fue de $4.3955 \mathrm{~g} / \mathrm{ml}$, 
presentando una diferencia del $62.79 \%$. Así, como se analizó para el cuerpo de la lata, al ser la base una de las partes que mayor resistencia mecánica debe poseer y al observar una densidad tan alta, puede decirse que su dureza también será alta y que estas partes bastante densas son las que menor porcentaje de aluminio tienen, pues se encuentran aleadas con mayores cantidades de cobre y magnesio que les confieren dichas propiedades.

Se observa, entonces, una clara diferencia entre la densidad promedio de las piezas elaboradas y las de las latas de las cuales estas provinieron. Esta diferencia es una prueba de que, si bien las latas de aluminio utilizadas no fueron puras debido a que se encuentran fabricadas con una aleación de aproximadamente un $97 \%$ de aluminio (según la teoría), durante el proceso de fundición se logró aumentar la pureza del aluminio. No obstante, es de notar que la pintura y capa de óxidos pudo influir de forma apreciable en esta diferencia de densidades tan notoria y que al eliminarse de forma casi completa estos componentes, durante el proceso de reciclaje, se haya obtenido una mayor concentración de aluminio puro en las piezas fabricadas.

En el gráfico 4 se muestra la influencia del azufre en la dureza de la aleación de aluminio. Así, a medida que se aumenta la concentración de azufre en la aleación, la dureza es mayor. Se observa que la dureza aumenta en 2.5 unidades con azufre al 1 $\%$ y que se incrementa en 9.6 unidades con la adición de azufre al $4.98 \%$.

En el gráfico 5 se observa cómo la presencia del vidrio influye de forma negativa en la dureza de la aleación. Con la adición de vidrio molido al $5.06 \%$ puede verse que la dureza de la aleación cambia de 50.8 a 34.6; por tanto, disminuye en 16.2 unidades. Esta es una pérdida elevada de dureza, incluso mayor que la ganancia de dureza que se obtuvo con la adición de azufre. Este hecho puede explicarse teniendo en cuenta que el vidrio posee un punto de fusión elevado de $1500^{\circ} \mathrm{C}$ (dependiendo del tipo de vidrio) y que a las temperaturas que alcanzó el horno no fue capaz de fundirse y liberar el silicio de la forma deseada, por lo que sus partículas se incrustaron dentro de la matriz metálica provocando porosidad.

Estos resultados muestran claramente la influencia única del azufre y del vidrio en una pieza de aluminio. De este modo, se ve que cuando el azufre se encuentra presente al $4.98 \%$, le confiere mayor dureza a la pieza; por el contrario, cuando el vidrio se encuentra casi en la misma cantidad (5.06 \%), ocurre un fenómeno muy diferente: la dureza de la pieza disminuye en gran medida. 
Los efectos negativos del vidrio no solamente se reflejan con estas dos pruebas. También se observa en la tabla 1 que para la fabricación de la pieza 1B se añadió sal de mesa al $2.02 \%$ y vidrio al $1.02 \%$. Al medir la dureza Brinell de esta pieza, se obtuvo un resultado de $55.37 \mathrm{HB}$; este valor de dureza disminuyó en 13.44 unidades para la pieza $2 \mathrm{~B}$, en la que se aumentó la concentración de vidrio, siendo este agregado al $4.96 \%$. A pesar de que para esta prueba también se varió la cantidad de sal de mesa utilizada, la diferencia solamente fue de $0.46 \%$, por lo que la disminución de la dureza se debe en su mayor parte al incremento en la concentración del vidrio.

También se debe notar la influencia que tuvo la adición de bicarbonato de sodio en la dureza de la aleación (ver gráfico 6), en donde se nota que hubo un incremento en la dureza, sin embargo, no fue alto, ya que correspondió a 1.9 unidades.

Es importante hacer un intento de dilucidar la influencia que tuvo la sal como fundente durante el proceso. Comparando, en la tabla 1, las durezas entre las piezas $2 \mathrm{~B}$ y $3 \mathrm{~B}$ se observa que, para casi una misma concentración de vidrio (4.96 \% para 2B y 5.06 $\%$ para 3B), la adición de sal resultó en un aumento de 7.33 en la dureza de la aleación. De esta amanera se observa que sin la adición del fundente esta fue de 34.6 y que al añadir la sal de mesa al $2.48 \%$ aumentó a $41.93 \mathrm{HB}$. Se calcula que la adición de sal de mesa a la aleación de aluminio y vidrio al 4.96 causó un aumento del $21.18 \%$ en la dureza de la pieza.

A pesar de los efectos negativos que el vidrio causa a una aleación de aluminio, es posible utilizar vidrio en una baja cantidad $(2.02 \%)$ y contrarrestar en gran parte estos efectos con la adición de sal de mesa al $1.02 \%$. Este procedimiento causará un aumento del $60.03 \%$ en la dureza del material.

Durante el proceso de fundición se logró un alto grado de recuperación de aluminio puro a partir de las latas. Esto se pudo comprobar con las mediciones de densidad, en las cuales se observa que la densidades de las piezas fundidas, en promedio, son mucho más cercanas a la teórica del aluminio $(2.7 \mathrm{~g} / \mathrm{ml})$ que la densidad promedio de las latas utilizadas. La densidad promedio de las piezas fundidas fue de $2.59 \mathrm{~g} / \mathrm{ml}$ y la de la lata fue de $3.08 \mathrm{~g} / \mathrm{ml}$.

Finalmente, cabe mencionar que algunas de las sustancias añadidas para fabricar las aleaciones sufren cambios químicos con el calor, descomponiéndose durante el proceso y que, técnicamente, las propiedades que le confieren a la misma se deben a los productos de dicha descomposición. 
Así, el bicarbonato de sodio se descompone en carbonato de sodio, dióxido de carbono y agua a $270{ }^{\circ} \mathrm{C}$, en donde el carbonato de sodio es el compuesto sólido que permanece en la aleación después de la descomposición.

\section{Tabla 1. Determinación de algunas propiedades de las piezas de aluminio elaboradas.}

\begin{tabular}{|c|c|c|c|}
\hline Pieza & Composición & $\begin{array}{c}\text { Densidad } \\
(\mathrm{g} / \mathrm{ml})\end{array}$ & $\begin{array}{c}\text { Dureza Brinell } \\
(\mathrm{HB})\end{array}$ \\
\hline $\mathrm{AB}$ & $100 \%$ Aluminio* & 2.3200 & 50.8 \\
\hline 1B & $\begin{array}{c}96.96 \% \text { Aluminio* } 2.02 \% \text { sal de mesa } \\
\mathrm{y} 1.02 \% \text { vidrio. }\end{array}$ & 2.6400 & 55.37 \\
\hline 2B & $\begin{array}{c}92.56 \% \text { Aluminio* } 4.96 \% \text { vidrio y } \\
2.48 \% \text { sal de mesa. }\end{array}$ & 2.6500 & 41.93 \\
\hline 3B & $94.94 \%$ Aluminio, $5.06 \%$ vidrio. & 2.5400 & 34.6 \\
\hline 4B & $99 \%$ Aluminio* $1 \%$ Azufre & 2.7200 & 53.3 \\
\hline 5B & $\begin{array}{c}94.94 \% \text { Aluminio* } 5.06 \% \text { bicarbonato } \\
\text { de sodio. }\end{array}$ & 2.6200 & 52.7 \\
\hline 6B & $95.02 \%$ Aluminio* $4.98 \%$ Azufre. & 2.6426 & 60.4 \\
\hline
\end{tabular}

"Se denomina "Aluminio" a la aleación obtenida del proceso de fundición, considerando que la mayor parte de las impurezas se perdieron durante el proceso.

(a) Para una carga de $62.5 \mathrm{Kgf}$ y un diámetro de bola de $5 \mathrm{~mm}$. 
Gráfico 4. Influencia de la concentración de azufre en la dureza de una aleación de aluminio

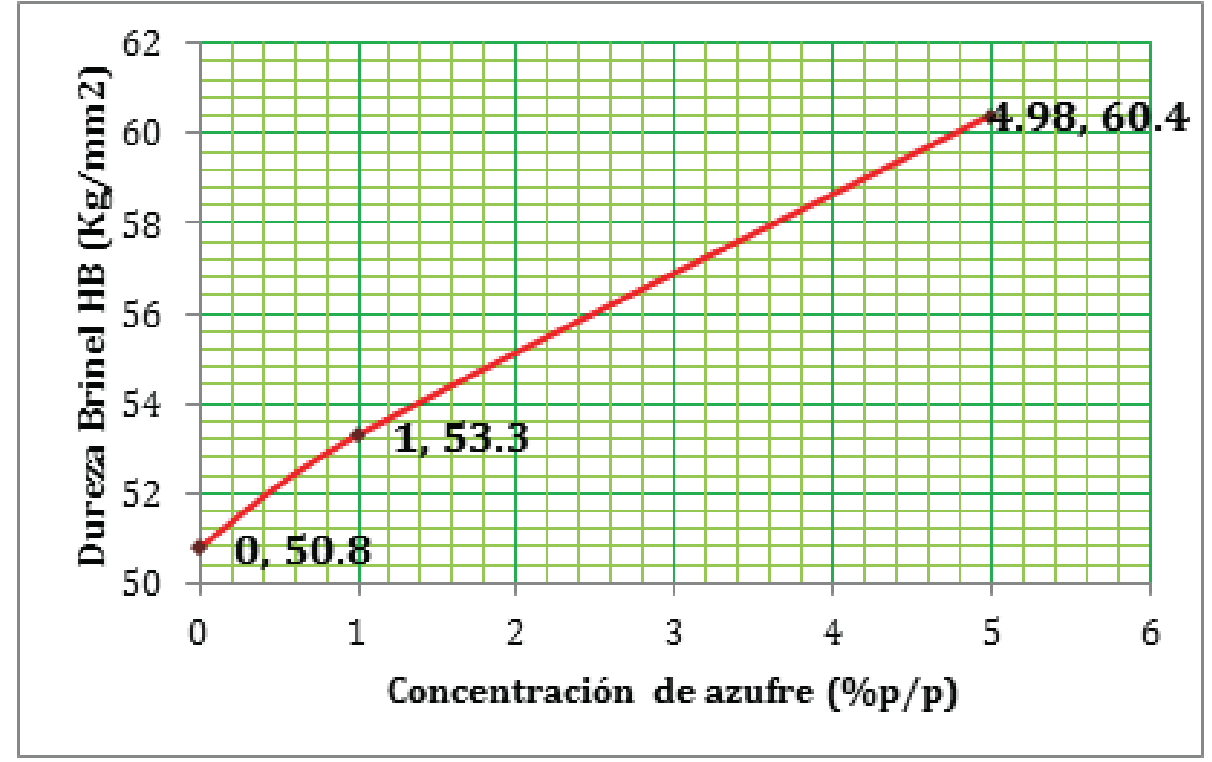

Gráfico 5. Influencia de la concentración de vidrio en la dureza de una aleación de aluminioo

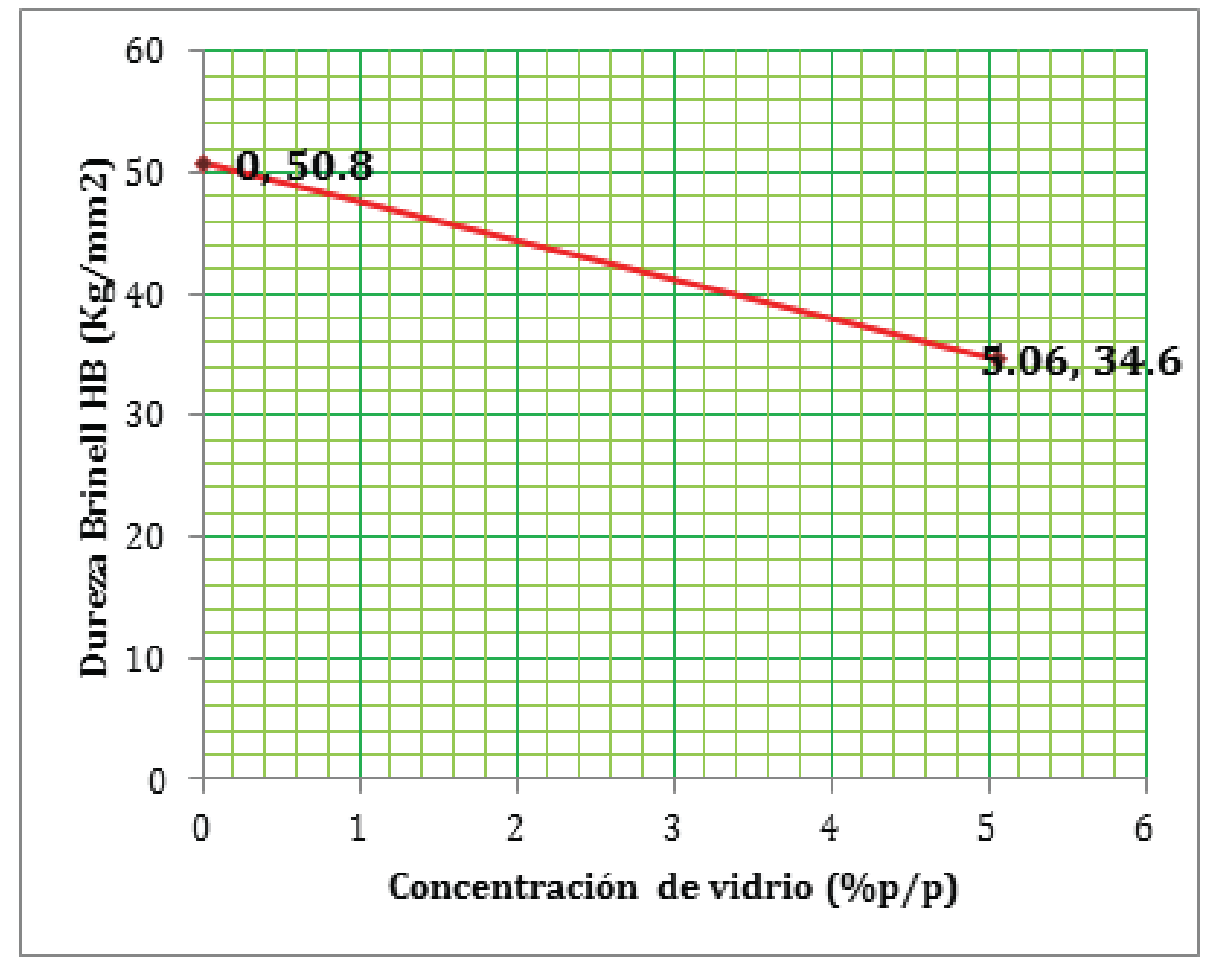


Gráfico 6. Influencia de la concentración de bicarbonato de sodio en la dureza de una aleación de aluminio

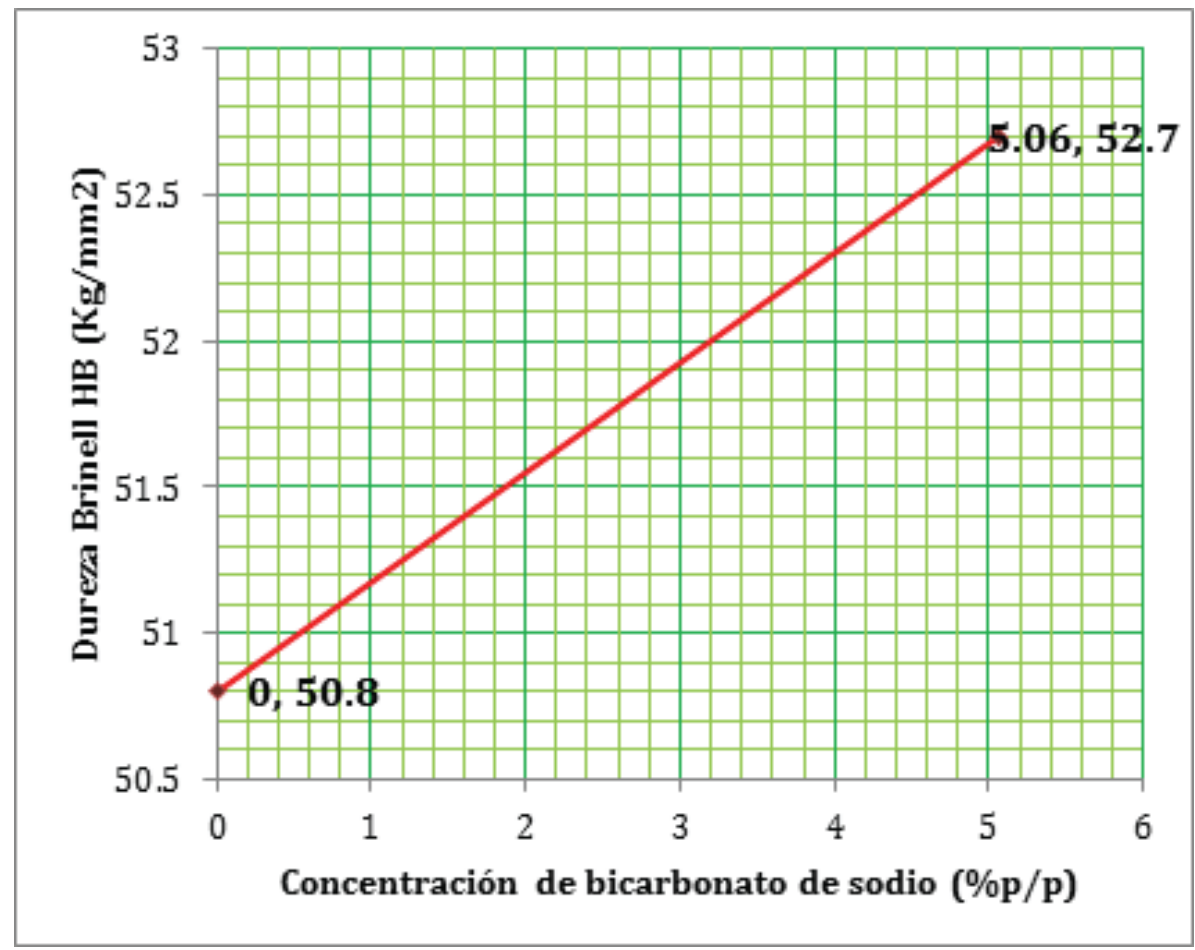

\section{CONCLUSIONES}

1. La presencia de azufre en concentraciones del $4.98 \%$ es beneficiosa para la aleación de aluminio, ya que incrementa su dureza en un $18.9 \%$. Además, puede decirse que, en un rango de concentración de $0 \%$ a $4.98 \%$ p/p de azufre, el grado de dureza en la aleación aumenta con la concentración de azufre.

2. La adición del vidrio en una matriz de aluminio ocasiona porosidad en la pieza, ya que provoca incrustación de partículas de vidrio en la matriz metálica, aumentando el grado de porosidad de la misma Por lo tanto, según los resultados obtenidos, puede concluirse que a mayor concentración de vidrio, menor dureza tendrá la aleación. Así, el vidrio no es un elemento adecuado para obtener una aleación de aluminio con mayor dureza. 
3. La presencia del bicarbonato de sodio no causa un aumento en la porosidad del aluminio, sin embargo, a diferencia del azufre, este componente no es el adecuado para aumentar la dureza en mayor grado.

4. La utilización de sales de metales alcalinos $(\mathrm{KCl}, \mathrm{NaCl}, \mathrm{KI})$ como fundentes contribuye a disminuir la porosidad de la aleación, ya que incrementa su dureza; por lo tanto, estas sales son un fundente eficaz para la fundición de aluminio.

5. Es posible obtener un alto grado de recuperación de aluminio puro a partir de latas de bebidas comercializadas en Honduras utilizando un horno de fundición impulsado por carbón vegetal.

\section{AGRADECIMIENTOS}

Al Instituto Nacional de Formación Profesional (INFOP), por las facilidades brindadas para usar su equipo de laboratorio en el desarrollo de esta investigación.

A la Ing. y Máster Covadonga Álvarez por su asesoría y motivación.

A todo el equipo de investigación del módulo de Metalurgia Aplicada de la Carrera de Química Industrial de la UNAH-VS del primer período de 2014 cuyos integrantes fueron:

Krizia Menjivar, Ana Rodríguez, Yelba Banegas, Ángela Jiménez, Reynaldo Enamorado, Carlos Pereira, Andrea Bardales.

\section{BIBLIOGRAFÍA}

Askeland, Donald y Phule, Pradeep. (2004). Ciencia e ingeniería de los materiales. México: Thompson Editores.

Groover, Mikell. (1997). Fundamentos de manufactura moderna. México: Editorial Prentice-Hall Hispanoamericana.

Sánchez, María. (2010). Tecnología de los materiales. México: Editorial Trillas.

William D., Callister Jr. (2007). Introducción a la ciencia e ingeniería de los materiales.

España: Editorial Reverté. 
DeGarmo, E.P., Black, J.T., Kohser, R.A., (2002) Materiales y Procesos de Fabricación. Barcelona, España: Editorial Reverté. 1. 223.

UNICAN. (2009) Aluminio y sus aleaciones. Cantabria, España. Departamento de Transportes, Tecnología de Proyectos y Procesos. Recuperado de: http://grupos.unican.es/gidai/web/asignaturas/CI/Aluminio.pdf

LINCOLN ELECTRIC. (2008) Soldeo de Aluminio y sus Aleaciones. Departamento de Formación Lincoln-KD S.A. Toledo, España. SOLYSOL. Recuperado de: http://solysol.com.es/data/documents/soldaduraaluminio.doc.pdf

IES Villalba Hervás. (2010)Tipos de Materiales. Tecnología Industrial I. Santa Cruz de Tenerife, España. Recuperado de: http://iesvillalbahervastecnologia.files.wordpress.com/2010/01/materiales_metales.pdf

UPV. (2010).Aleaciones para Ingeniería (2010). Curso de Fundamentos de la Ciencia de Materiales. Valencia, España. Recuperado de: http://www.upv.es/materiales/Fcm/Fcm13/fcm13_4.html

IES Villalba Hervás. (2010) Tipos de Materiales. Tecnología Industrial I. Santa Cruz de Tenerife, Recuperado de: http://iesvillalbahervastecnologia.files.wordpress.com/2010/01/materiales_metales.pdf

ABOOD, Kiffaya. (2008) Recycling of Aluminum Beverage Cans. Journal of Engineering and Development. Baghdad, Iraq. 12 (3). Recuperado de: http://www.iasj.net/iasj?func=fulltext\&ald=10274

Departament de Territori y Sostenibilitat. (2008) Reciclaje del Aluminio Generalitat de Catalunya. Amigos de La Tierra. España. Recuperado de: http://mediambient.gencat.cat/web/.content/home/ambits_dactuacio/educacio_i_sostenibilitat/ educacio_per_a_la_sostenibilitat/suport_educatiu/recursos_educatius/residus/recialu/reciclatge_de___alumini.pdf 